\title{
Constructions of Round Fold Maps on Smooth Bundles
}

\author{
Naoki KITAZAWA \\ Tokyo Institute of Technology \\ (Communicated by Y. Kuno)
}

\begin{abstract}
In this paper, we construct round fold maps or stable fold maps with singular value sets of concentric spheres introduced by the author [11] on smooth bundles over spheres and bundles over more general manifolds. The class of round fold maps includes some special generic maps on spheres (see [20] for example) and such maps have been constructed on smooth bundles over the standard sphere $S^{k}$ with $k \geq 2$ and connected sums of smooth bundles over $S^{k}$ with $k \geq 2$ with fibers diffeomorphic to standard spheres, for example, in previous studies by the author ([10], [12]). In this paper, we obtain round fold maps which do not appear in these studies with information on the diffeomorphism types of their source manifolds in new manners.
\end{abstract}

\section{Introduction}

Fold maps are fundamental tools in generalizing the theory of Morse functions. Studies of such maps were started by Whitney ([25]) and Thom ([24]) in the 1950's. A fold map from an $m$-dimensional smooth manifold into an $n$-dimensional smooth manifold without boundary is defined as a smooth map whose singular points are of the form

$$
\left(x_{1}, \ldots, x_{m}\right) \mapsto\left(x_{1}, \ldots, x_{n-1}, \sum_{j=n}^{m-i} x_{j}^{2}-\sum_{j=m-i+1}^{m} x_{j}^{2}\right)
$$

for an integer $0 \leq i \leq m-n+1(m \geq n \geq 1)$. A Morse function is regarded as a fold map $(n=1)$. For such a fold map, the followings hold.

1. The singular set, or the set of all the singular points, is a closed smooth submanifold of dimension $n-1$ of the source manifold.

2. The restriction map to the singular set is a smooth immersion of codimension 1. Special generic maps are defined as fold maps whose singular points are of the form

$$
\left(x_{1}, \ldots, x_{m}\right) \mapsto\left(x_{1}, \ldots, x_{n-1}, \sum_{j=n}^{m} x_{j}^{2}\right)
$$

Received July 16, 2013; revised December 10, 2013

Mathematics Subject Classification: 57R45 (Primary), 57N15 (Secondary)

Key words and phrases: Singularities of differentiable maps; singular sets, fold maps. Differential topology 
and they are studied in [3], [8], [19], [21] and [22] for example.

In [11], round fold maps, which will be mainly studied in this paper, were introduced. A round fold map is defined as a fold map satisfying the followings.

1. The singular set is a disjoint union of standard spheres.

2. The restriction map to the singular set is an embedding.

3. The singular value set is a disjoint union of spheres embedded concentrically.

For example, some special generic maps on spheres are examples of round fold maps. Any $m$-dimensional standard sphere admits such a map into $\mathbf{R}^{n}$ with $m \geq n \geq 2$ and any smooth manifold homeomorphic to a sphere whose dimension is larger than 1 and not 4 admits such a map into the plane (see also [20]).

In [11], homology groups and homotopy groups of manifolds admitting round fold maps are studied. Now, how about the homeomorphism and the diffeomorphism types of the source manifolds? As answers, some examples of round fold maps and the diffeomorphism types of their source manifolds are given by the author in [10] and [12]. For example, we have obtained round fold maps on smooth bundles over $S^{n}$ with $n \geq 2$ and $m$-dimensional closed smooth manifolds represented as connected sums of families of smooth bundles over $S^{n}$ with fibers diffeomorphic to $S^{m-n}$ and $m$-dimensional spheres admitting round fold maps with connected singular sets into $\mathbf{R}^{n}$ with $n \geq 2$ and $m \geq 2 n$ assumed. In this paper, as new answers to the question, we construct new round fold maps on closed manifolds having the structures of smooth bundles over (exotic) spheres and bundles over more general manifolds.

This paper is organized as the following.

Section 2 is for preliminaries. We recall fold maps. We also recall special generic maps and simple fold maps. Finally we review the Reeb space of a smooth map, which is defined as the space consisting of all the connected components of all the fibers of the smooth map.

In section 3, we recall round fold maps and some terminologies on round fold maps such as axes and proper cores. We also recall a $C^{\infty}$ trivial round fold map. We introduce results on the diffeomorphism types of manifolds admitting $C^{\infty}$ trivial round fold maps shown by the author in [10] and [12] (Proposition 2).

In section 4, we give some new examples of round fold maps from manifolds having the structure of smooth bundles. First, we construct new round fold maps on closed smooth manifolds having the structures of smooth bundles over (exotic) spheres (Theorem 1). Next, we introduce a locally $C^{\infty}$ trivial round fold map as a map satisfying a kind of triviality around the connected components of the singular value set and define P-operations. We apply these operations and the theory of $S^{1}$-bundles to construct families of new round fold maps on smooth $S^{1}$-bundles over the source manifolds of locally smoothly trivial round fold maps in Theorems 3 and 4.

Throughout this paper, we assume that $M$ is a closed smooth manifold of dimension $m$, that $N$ is a smooth manifold of dimension $n$ without boundary, that $f: M \rightarrow N$ is a smooth map and that $m \geq n \geq 1$ holds. We denote the singular set of $f$, or the set consisting of all the singular points of $f$, by $S(f)$.

All manifolds and smooth maps are of class $C^{\infty}$ unless otherwise stated in the proceeding 
sections.

The author would like to express his gratitude to Mitsutaka Murayama, Osamu Saeki, Kazuhiro Sakuma and Takahiro Yamamoto for helpful comments and constant encouragement. The author also thanks the referee for useful comments, which improved this paper.

\section{Preliminaries}

2.1. Fold maps. First, we recall fold maps, which are simplest generalizations of Morse functions. For precise information, see [9], [15] and [16] for example.

Definition 1. For a map $f: M \rightarrow N$, a point $p \in M$ is said to be a fold point of $f$ if at $p$, the map $f$ has the normal form

$$
f\left(x_{1}, \ldots, x_{m}\right):=\left(x_{1}, \ldots, x_{n-1}, \sum_{j=n}^{m-i} x_{j}^{2}-\sum_{j=m-i+1}^{m} x_{j}^{2}\right)
$$

and $f$ is said to be a fold map if all the singular points of $f$ are fold.

If a point $p \in M$ is a fold point of $f$, then we can define $j:=\min \{i, m-n+1-i\}$ uniquely in the previous definition. We call $p$ a fold point of index $j$ of $f$. We call a fold point of index 0 a definite fold point of $f$ and we call $f$ a special generic map if all the singular points are definite fold points. For special generic maps, see [3], [8], [20] and [22] for example. Let $f$ be a fold map. Then, the singular set $S(f)$ and the set of all the fold points whose indices are $i$ (we denote the set of all such points by $\left.F_{i}(f)\right)$ are $(n-1)$-dimensional submanifolds of $M$. The restriction map $\left.f\right|_{S(f)}$ is an immersion.

A Morse function on a closed manifold is naturally regarded as a fold map $(n=1)$. A Morse function on a closed manifold which has just two singular points is naturally regarded as a special generic map.

2.2. Reeb spaces. We review the Reeb space of a map.

Definition 2. Let $X, Y$ be topological spaces. For $p_{1}, p_{2} \in X$ and for a map $c$ : $X \rightarrow Y$, we define as $p_{1} \sim_{c} p_{2}$ if and only if $p_{1}$ and $p_{2}$ are in the same connected component of $c^{-1}(p)$ for some $p \in Y$. The relation $\sim_{c}$ is an equivalence relation.

We denote the quotient space $X / \sim_{c}$ by $W_{c}$ and call it the Reeb space of $c$.

We denote the induced quotient map from $X$ into $W_{c}$ by $q_{c}$. We define $\bar{c}: W_{c} \rightarrow Y$ so that $c=\bar{c} \circ q_{c}$. $W_{c}$ is often homeomorphic to a polyhedron.

Here, we introduce terms on spheres and fiber bundles which are important in this paper.

A smooth sphere means a manifold homeomorphic to a sphere. An almost-sphere of dimension $k \geq 1$ means a sphere given by glueing two $k$-dimensional closed standard discs together by a diffeomorphism between the boundaries.

We often use terminologies on (fiber) bundles in this paper (see also [23]). For a topological space $X$, an $X$-bundle is a bundle whose fiber is $X$. A bundle whose structure group 
is $G$ is said to be a trivial bundle if it is equivalent to the product bundle as a bundle whose structure group is $G$. Especially, a trivial bundle whose structure group is a subgroup of the homeomorphism group of the fiber is said to be a topologically trivial bundle. In this paper, a smooth (PL) bundle means a bundle whose fiber is a smooth (resp. PL) manifold and whose structure group is a subgroup of the diffeomorphism group (resp. PL homeomorphism group) of the fiber. A linear bundle is a smooth bundle whose fiber is a standard disc or a standard sphere and whose structure group is a subgroup of an orthogonal group.

The following Proposition 1 is well-known and we omit the proof. See [13] and [19] for example. We often use these statements implicitly in the proceeding sections.

PROPOSITION 1. Let $f: M \rightarrow N$ be a special generic map or a simple fold map or a stable fold map from a closed manifold $M$ of dimension $m$ into a manifold $N$ of dimension $n$. Then $W_{f}$ has the structure of a polyhedron and the followings hold.

1. $W_{f}-q_{f}(S(f))$ is uniquely given the structure of a manifold such that $\left.q_{f}\right|_{M-S(f)}$ : $M-S(f) \rightarrow W_{f}-q_{f}(S(f))$ is a submersion. Furthermore, for any compact smooth submanifold $R$ of dimension $n$ of any connected component of $W_{f}-q_{f}(S(f)), R$ is a subpolyhedron of $W_{f}$ and $\left.q_{f}\right|_{q_{f}^{-1}(R)}: q_{f}^{-1}(R) \rightarrow R$ gives the structure of a smooth bundle whose fiber is a connected smooth manifold of dimension $m-n$.

2. The restriction of $q_{f}$ to the set $F_{0}(f)$ of all the definite fold points is injective. Branched points of the Reeb space $W_{f}$ are in the image $q_{f}\left(F_{1}(f)\right)$ of the set of all the fold points whose indices are 1 . Points of $W_{f}$ which are not branched and which have open neighborhoods homeomorphic to open sets of $\mathbf{R}^{n}$ are in the set $W_{f}-q_{f}\left(F_{0}(f)\right)$.

3. $f$ is simple if and only if $\left.q_{f}\right|_{S(f)}: S(f) \rightarrow W_{f}$ is injective.

4. If $f$ is simple, then for any connected component $C$ of $S(f), q_{f}(C)$ has a small regular neighborhood $N\left(q_{f}(C)\right)$ in $W_{f}$ such that $q_{f}^{-1}\left(N\left(q_{f}(C)\right)\right)$ has the structure of a smooth bundle over $q_{f}(C)$.

Furthermore, assume that $N\left(q_{f}(C)\right)$ does not contain branched points and that $N\left(q_{f}(C)\right)-q_{f}(C)$ is not connected, then $N\left(q_{f}(C)\right)$ has the structure of a trivial PL $[-1,1]$-bundle over $q_{f}(C)$ and $q_{f}(C)$ corresponds to the 0-section. $q_{f}^{-1}\left(N\left(q_{f}(C)\right)\right)$ has the structure of a smooth bundle over $q_{f}(C)$ and the bundle structure is given by the composition of $\left.q_{f}\right|_{q_{f}^{-1}\left(N\left(q_{f}(C)\right)\right)}: q_{f}^{-1}\left(N\left(q_{f}(C)\right)\right) \rightarrow$ $N\left(q_{f}(C)\right)$ and the projection to $q_{f}(C)$.

5. For any connected component $C$ of $F_{0}(f)$, any small regular neighborhood of $q_{f}(C)$ has the structure of a trivial PL [0,1]-bundle over $q_{f}(C)$ such that $q_{f}(C)$ corresponds to the 0-section. We can take a small regular neighborhood $N\left(q_{f}(C)\right)$ of $q_{f}(C)$ so that $q_{f}^{-1}\left(N\left(q_{f}(C)\right)\right)$ has the structure of a linear $D^{m-n+1}$-bundle over $q_{f}(C)$. More precisely, the bundle structure is given by the composition of $\left.q_{f}\right|_{q_{f}^{-1}\left(N\left(q_{f}(C)\right)\right.}: q_{f}^{-1}\left(N\left(q_{f}(C)\right)\right) \rightarrow N\left(q_{f}(C)\right)$ and the projection to $q_{f}(C)$.

6. Let $f$ be simple and $m-n \geq 1$. If $m-n=1$, then we also assume that $M$ is 
orientable.

Then for any connected component $C$ of the set $F_{1}(f)$ of all the fold points whose indices are 1 such that for any point $p \in q_{f}(C)$, any small neighborhood of $N(p)$ of $p$ and any point $q$ in $N(p)-q_{f}(C)$, the inverse image $q_{f^{-1}}(q)$ is an almostsphere, any small regular neighborhood $N\left(q_{f}(C)\right)$ of $q_{f}(C)$ has the structure of a $K$-bundle over $q_{f}(C)$ where $K:=\left\{r \exp (2 \pi i \theta) \in \mathbf{C} \mid 0 \leq r \leq 1, \theta=0, \frac{1}{3}, \frac{2}{3}\right\}$ with the structure group consisting of just two elements where one element is defined as the identity transformation and the other element is defined as the transformation defined by $z \rightarrow \bar{z}(\bar{z} \in K$ is the complex conjugation of $z \in K)$ such that $q_{f}(C)$ corresponds to the 0-section. We can take the neighborhood $N\left(q_{f}(C)\right)$ of $q_{f}(C)$ so that $q_{f}^{-1}\left(N\left(q_{f}(C)\right)\right)$ has the structure of a $C^{\infty}$ bundle over $q_{f}(C)$ with fibers $P L$ homeomorphic to $S^{m-n+1}$ with the interior of a union of disjoint three $(m-n+1)$ dimensional standard closed discs removed. More precisely, the bundle structure is given by the composition of $\left.q_{f}\right|_{q_{f}^{-1}\left(N\left(q_{f}(C)\right)\right)}: q_{f}^{-1}\left(N\left(q_{f}(C)\right)\right) \rightarrow N\left(q_{f}(C)\right)$ and the projection to $q_{f}(C)$.

\section{Round fold maps}

In this section, we review round fold maps. See also [11].

First we recall $C^{\infty}$ equivalence (see also [9] for example). For two $C^{\infty}$ maps $f_{1}: X_{1} \rightarrow$ $Y_{1}$ and $f_{2}: X_{2} \rightarrow Y_{2}$, we say that they are $C^{\infty}$ equivalent if there exist diffeomorphisms $\phi_{X}: X_{1} \rightarrow X_{2}$ and $\phi_{Y}: Y_{1} \rightarrow Y_{2}$ such that the following diagram commutes.

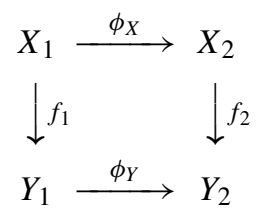

Definition 3 (round fold maps ([11])). A map $f: M \rightarrow \mathbf{R}^{n}(m \geq n \geq 2$ ) is said to be a round fold map if $f$ is $C^{\infty}$ equivalent to a fold map $f_{0}: M_{0} \rightarrow \mathbf{R}^{n}$ on a closed $C^{\infty}$ manifold $M_{0}$ such that the followings hold.

1. The singular set $S\left(f_{0}\right)$ is a disjoint union of $(n-1)$-dimensional standard spheres and consists of $l \in \mathbf{N}$ connected components.

2. The restriction map $\left.f_{0}\right|_{S\left(f_{0}\right)}$ is an embedding.

3. Let $D_{r}^{n}:=\left\{\left(x_{1}, \ldots, x_{n}\right) \in \mathbf{R}^{n} \mid \sum_{k=1}^{n} x_{k}^{2} \leq r\right\}$. Then, $f_{0}\left(S\left(f_{0}\right)\right)=\sqcup_{k=1}^{l} \partial D_{k}^{n}$ holds.

We call $f_{0}$ a normal form of $f$. We call a ray $L$ from $0 \in \mathbf{R}^{n}$ an axis of $f_{0}$ and $D^{n}{ }_{\frac{1}{2}}$ the proper core of $f_{0}$. Suppose that for a round fold map $f$, its normal form $f_{0}$ and diffeomorphisms $\Phi: M \rightarrow M_{0}$ and $\phi: \mathbf{R}^{n} \rightarrow \mathbf{R}^{n}$, the relation $\phi \circ f=f_{0} \circ \Phi$ holds. Then, for an 


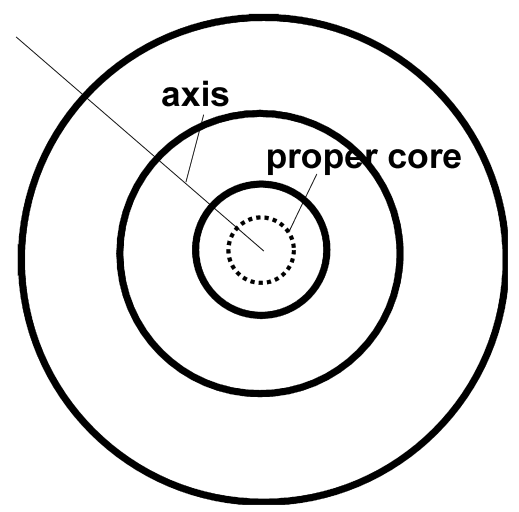

FIGURE 1. An axis and a proper core of a round fold map (Definition 4)

axis $L$ of $f_{0}$, we also call $\phi^{-1}(L)$ an axis of $f$ and for the proper core $D^{n}{ }_{\frac{1}{2}}$ of $f_{0}$, we also call $\phi^{-1}\left(D_{\frac{1}{2}}^{n}\right)$ a proper core of $f$.

Let $f: M \rightarrow \mathbf{R}^{n}$ be a round fold map and let $L$ be its axis.

Let $f$ be a normal form of a round fold map and let $P_{1}:=D^{n}{ }_{\frac{1}{2}}$. We set $E:=f^{-1}\left(P_{1}\right)$ and $E^{\prime}:=M-f^{-1}\left(\operatorname{Int} P_{1}\right)$. We set $F:=f^{-1}(p)$ for $p \in \partial P_{1}$. We put $P_{2}:=\mathbf{R}^{n}-\operatorname{Int} P_{1}$. Let $f_{1}:=\left.f\right|_{E}: E \rightarrow P_{1}$ if $F$ is non-empty and let $f_{2}:=\left.f\right|_{E^{\prime}}: E^{\prime} \rightarrow P_{2}$.

$f_{1}$ gives the structure of a trivial smooth bundle over $P_{1}$ and $\left.f_{1}\right|_{\partial E}: \partial E \rightarrow \partial P_{1}$ gives the structure of a trivial smooth bundle over $\partial P_{1}$ if $F$ is non-empty. $\left.f_{2}\right|_{\partial E^{\prime}}: \partial E^{\prime} \rightarrow \partial P_{2}$ gives the structure of a trivial smooth bundle over $\partial P_{2}$.

We can give $E^{\prime}$ the structure of a smooth bundle over $\partial P_{2}$ as follows.

Since for $\pi_{P}(x):=\frac{1}{2} \frac{x}{|x|}\left(x \in P_{2}\right),\left.\pi_{P} \circ f\right|_{E^{\prime}}$ is a proper submersion, this map gives $E^{\prime}$ the structure of a smooth $f^{-1}(L)$-bundle over $\partial P_{2}$ (apply Ehresmann's fibration theorem [5]).

For a round fold map $f$ which is not a normal form, we can consider similar maps and bundles.

Definition 4 ([11]). Let $f: M \rightarrow \mathbf{R}^{n}$ be a round fold map $(n \geq 2)$. If the projection from the total space of the bundle $E^{\prime}$ onto the base space $\partial P_{2}\left(\partial P_{2}\right.$ is diffeomorphic to $S^{n-1}$ ) introduced above gives the structure of a smooth trivial bundle, then $f$ is said to be $C^{\infty}$ trivial.

We can construct a $C^{\infty}$ trivial round fold map as in the following manner, which was introduced in [11].

Before the construction, we introduce good Morse functions on compact manifolds possibly with non-empty boundaries. A Morse function on a compact manifold with non-empty 
boundary is said to be good if on the boundary, it is constant and minimal, singular points of it are not on the boundary and at two distinct singular points, the singular values are always distinct. A Morse function on a closed, or compact manifold without boundary is said to be good if at two distinct singular points, the singular values are always distinct.

Let $\bar{M}$ be a compact manifold with non-empty boundary $\partial \bar{M}$. Then, there exists a good Morse function $\tilde{f}: \bar{M} \rightarrow[a,+\infty)$, where $a$ is the minimal value.

Let $\Phi: \partial\left(\bar{M} \times \partial\left(\mathbf{R}^{n}-\operatorname{Int} D^{n}\right)\right) \rightarrow \partial\left(\partial \bar{M} \times D^{n}\right)$ and $\phi: \partial\left(\mathbf{R}^{n}-\operatorname{Int} D^{n}\right) \rightarrow \partial D^{n}$ be diffeomorphisms. Let $p_{1}: \partial \bar{M} \times \partial\left(\mathbf{R}^{n}-\operatorname{Int} D^{n}\right) \rightarrow \partial\left(\mathbf{R}^{n}-\operatorname{Int} D^{n}\right)$ and $p_{2}: \partial \bar{M} \times \partial D^{n} \rightarrow$ $\partial D^{n}$ be the canonical projections. Suppose that the following diagram commutes.

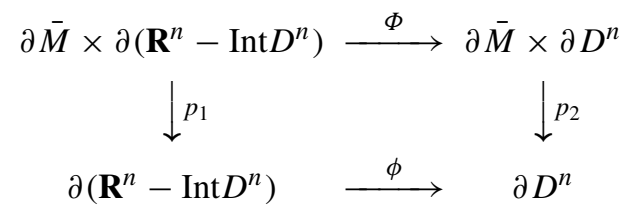

By using $\Phi$, we construct $M:=\left(\partial \bar{M} \times D^{n}\right) \bigcup_{\Phi}\left(\bar{M} \times \partial\left(\mathbf{R}^{n}-\operatorname{Int} D^{n}\right)\right)$. Let $p: \partial \bar{M} \times$ $D^{n} \rightarrow D^{n}$ be the canonical projection. Then, by gluing the maps $p$ and $\tilde{f} \times \operatorname{id}_{S^{n-1}}$ together by the diffeomorphisms $\Phi$ and $\phi$, we have a round fold map $f:=p \bigcup_{\Phi, \phi}\left(\tilde{f} \times \mathrm{id}_{S^{n-1}}\right)$ whose source manifold is $M$.

If $\bar{M}$ is a compact manifold without boundary, then there exists a good Morse function $\tilde{f}: \bar{M} \rightarrow[a,+\infty)$ such that $\tilde{f}(\bar{M}) \subset(a,+\infty)$. We are enough to consider the map $\tilde{f} \times \operatorname{id}_{S^{n-1}}$ and embed $[a,+\infty) \times S^{n-1}$ into $\mathbf{R}^{n}$ to construct a round fold map whose source manifold is $\bar{M} \times S^{n-1}$.

From now on, a bundle means a smooth bundle unless otherwise stated. First, we introduce Theorem 1 of [12].

Proposition 2 ([12]). Let $M$ be a closed manifold of dimension $m$. Let $n \in \mathbf{N}$ and $m \geq n \geq 2$.

1. Let $M$ have the structure of a bundle over $S^{n}$ whose fiber is a closed manifold $F(\neq \emptyset)$. Then, there exists a $C^{\infty}$ trivial round fold map $f: M \rightarrow \mathbf{R}^{n}$ such that the fiber of a point in a proper core of $f$ is diffeomorphic to a disjoint union of two copies of $F$ and that for an axis $L$ of $f, f^{-1}(L)$ is diffeomorphic to $F \times[0,1]$.

2. Suppose that a $C^{\infty}$ trivial round fold map $f: M \rightarrow \mathbf{R}^{n}$ exists and that for an axis $L$ of $f$ and a closed manifold $F$ of dimension $m-n, f^{-1}(L)$ is diffeomorphic to $F \times[-1,1]$. Then, $M$ has the structure of an $F$-bundle over $S^{n}$. 


\section{Round fold maps on bundles over spheres and constructions of round fold maps by P-operations}

In this section, we construct a round fold map on a bundle over a smooth sphere and a bundle over the source manifold of a locally $C^{\infty}$ trivial round fold map in new manners.

4.1. Round fold maps on bundles over spheres. First we review a round special generic group defined in [12]. Let $\Theta_{k}$ be the h-cobordism group of $k$-dimensional $(k \geq 2)$ smooth oriented spheres. It follows easily that the set of all the classes of $\Theta_{k_{1}}$ consisting of spheres admitting round fold maps with connected singular sets into $\mathbf{R}^{k_{2}}\left(k_{1} \geq k_{2} \geq 2\right)$ is a subgroup of $\Theta_{k_{1}}$. In fact, we are enough to consider the connected sum of given two round fold maps with connected singular sets (for the connected sum of given two special generic maps into Euclidean spaces, see section 5 of [19] for example). We denote the subgroup by $\Theta_{\left(k_{1}, k_{2}\right)} \subset \Theta_{k_{1}}$ and call it the $\left(k_{1}, k_{2}\right)$ round special generic group.

THEOREM 1. For any sphere $\Sigma$ in a class of $\Theta_{m, n}(m \geq n \geq 2)$ and any closed manifold $F \neq \varnothing$, any closed manifold $M$ having the structure of an $F$-bundle over $\Sigma$ admits a $C^{\infty}$ trivial round fold map into $\mathbf{R}^{n}$.

PROOF. $\quad \Sigma$ admits a round fold map $f: \Sigma \rightarrow \mathbf{R}^{n}$ whose singular set is connected and the map $f$ is $C^{\infty}$ trivial. Let $P$ be a proper core of $f$ and $Q$ be a small closed tubular neighborhood of the singular value set $f(S(f))$. We may assume that $\partial P \subset \partial Q$ holds. For a diffeomorphism $\phi$ which is regarded as an isomorphism between the two trivial $C^{\infty}$ bundles $\partial P \times S^{m-n}$ over $\partial P$ and $\partial P \times \partial D^{m-n+1}$ over $\partial P$ inducing the identification between the base spaces, $\Sigma$ is regarded as $\left(P \times S^{m-n}\right) \bigcup_{\phi}\left(\partial P \times D^{m-n+1}\right) . P \times S^{m-n} \subset \Sigma$ and $\partial P \times D^{m-n+1} \subset \Sigma$ are regarded as subspaces of contractible spaces $\Sigma-\left\{p_{1}\right\}$ and $\Sigma-$ $\left\{p_{2}\right\}\left(p_{1}, p_{2} \in \Sigma\right)$, respectively, so, if we restrict the base space of any $F$-bundle over $\Sigma$ to $P \times S^{m-n} \subset \Sigma$ and $\partial P \times D^{m-n+1} \subset \Sigma$, then the resulting bundles are trivial $C^{\infty}$ bundles. So, for every $F$-bundle over $\Sigma$, there exists a diffeomorphism $\Phi$ regarded as a bundle isomorphism between the two trivial $F$-bundles $\partial P \times S^{m-n} \times F$ over $\partial P \times S^{m-n}$ and $\partial P \times \partial D^{m-n+1} \times F$ over $\partial P \times \partial D^{m-n+1}$ inducing $\phi$ and the $C^{\infty} F$-bundle over $\Sigma$ is regarded as $\left(P \times S^{m-n} \times F\right) \bigcup_{\Phi}\left(\partial P \times D^{m-n+1} \times F\right)$.

We have a good Morse function $\tilde{f}: D^{m-n+1} \times F \rightarrow[a,+\infty)$, where $a$ is the minimum. By using the diffeomorphism $\Phi$ and the identification map in the target manifold $\mathbf{R}^{n}$, we can glue the canonical projection $p: P \times S^{m-n} \times F \rightarrow P$ and the map $\tilde{f} \times \operatorname{id}_{\partial P}$ to obtain a desired round fold map $f$ as mentioned after Definition 4. This completes the proof.

REMARK 1. Theorem 1 has been shown as a part of Theorem 1 of [12] or Proposition 2 of the present paper in the case where $\Sigma=S^{n}$ holds. In section 4 of [10], it has been shown in the case where $\Sigma=S^{n}$ holds with the assumption that $F$ is an almost-sphere and the resulting map satisfies all the conditions in Proposition 3 later and has the singular set with 
two connected components. The proof in the present paper can be regarded as an extension of the original proofs.

4.2. Locally $C^{\infty}$ trivial round fold maps and P-operations. First we introduce a locally $C^{\infty}$ trivial round fold map.

Definition 5. Let $f: M \rightarrow \mathbf{R}^{n}$ be a round fold map ( $\left.n \geq 2\right)$. Assume that for any connected component $C$ of $f(S(f))$ and a small closed tubular neighborhood $N(C)$ of $C$ such that $\partial N(C)$ is the disjoint union of two connected components $C_{1}$ and $C_{2}, f^{-1}(N(C))$ has the structures of trivial bundles over $C_{1}$ and $C_{2}$ and $\left.f\right|_{f^{-1}\left(C_{1}\right)}: f^{-1}\left(C_{1}\right) \rightarrow C_{1}$ and $\left.f\right|_{f^{-1}\left(C_{2}\right)}: f^{-1}\left(C_{2}\right) \rightarrow C_{2}$ give the structures of subbundles of the bundles $f^{-1}(N(C))$. Then, $f$ is said to be locally $C^{\infty}$ trivial. We call a fiber $F_{C}$ of the bundle $f^{-1}(N(C))$ a normal fiber of $C$ corresponding to the bundle $f^{-1}(N(C))$.

For example, the resulting round fold map constructed in the proof of Theorem 1 is locally $C^{\infty}$ trivial and $C^{\infty}$ trivial. We introduce results shown in [10] or [12] by using terms such as (locally) $C^{\infty}$ trivial maps.

Proposition 3 ([10], [12]). Let $m, n \in \mathbf{N}, n \geq 2$ and $m \geq 2 n$. Let $M$ be a connected sum of a finite number of $m$-dimensional closed manifolds having the structures of $S^{m-n}$-bundles over $S^{n}$ and an oriented manifold in a class of $\Theta_{(m, n)}$. Then, there exists a locally $C^{\infty}$ trivial round fold map $f: M \rightarrow \mathbf{R}^{n}$ such that the followings hold.

1. Regular fibers of $f$ are disjoint unions of standard spheres.

2. The number of connected components of the fiber of a point in a proper core of $f$ equals the number of connected components of $S(f)$.

3. For any connected component $C$ of $f(S(f))$ and a small closed tubular neighborhood $N(C)$ of $C, f^{-1}(N(C))$ has the structure of a trivial bundle over $C$ as in Definition 5 such that a normal fiber $F_{C}$ of $C$ corresponding to the bundle $f^{-1}(N(C))$ is diffeomorphic to a disjoint union of a finite number of the following manifolds.

(a) $D^{m-n+1}$.

(b) $S^{m-n+1}$ with the interior of a union of disjoint three $(m-n+1)$-dimensional standard closed discs removed.

We introduce the construction of a map satisfying all the conditions mentioned in Proposition 3, which was performed in [10] or [12].

Let $m$ and $n$ be integers satisfying the assumption of Proposition 3. Let $M_{1}$ and $M_{2}$ be manifolds represented as connected sums of finite numbers of $m$-dimensional closed manifolds having the structures of $S^{m-n}$-bundles over $S^{n}$ and oriented manifolds in some classes of $\Theta_{(m, n)}$. Assume that $M_{i}$ admits a round fold map $f_{i}: M_{i} \rightarrow \mathbf{R}^{n}$ satisfying the conditions for $i=1,2$.

Let $P_{1}$ be a proper core of $f_{1}$ and let $P_{2}$ be a small closed tubular neighborhood of the connected component of $f_{2}\left(S\left(f_{2}\right)\right)$ which is the boundary of $f_{2}\left(M_{2}\right)$ (see also FIGURE 2). 
Let $V_{1}$ be a connected component of $f_{1}^{-1}\left(P_{1}\right)$ such that $\left.f\right|_{V_{1}}: V_{1} \rightarrow P_{1}$ gives the structure of a trivial smooth $S^{m-n}$-bundle over $D^{n}$, which exists by the assumption on $f_{1}$, and let $V_{2}:=f_{2}^{-1}\left(P_{2}\right) . \quad V_{2}$ is a closed tubular neighborhood of $f_{2}^{-1}(C) \subset M_{2}$. The inclusion of $f_{2}{ }^{-1}(C)$ into $M_{2}$ is null-homotopic since $\pi_{n-1}\left(M_{2}\right) \cong\{0\}$ holds. From the inequality $m \geq 2 n=2(n-1)+2$, the inclusion of $f^{-1}(C)$ into $M_{2}$ is unknot in the $C^{\infty}$ category and $V_{2}$ has the structure of a trivial linear $D^{m-n+1}$-bundle. More precisely, $\left.f_{2}\right|_{\partial V_{2}}$ gives the structure of a subbundle of the bundle.

From the discussions above, we may regard that the following holds for a diffeomorphism $\Phi: \partial V_{2} \rightarrow \partial V_{1}$ regarded as a bundle isomorphism between the two trivial $S^{m-n+1}$ bundles over $S^{n-1}$ inducing a diffeomorphism between the base spaces and an orientation reversing diffeomorphism $\Psi: \partial D^{m} \rightarrow \partial D^{m}$ extending to a diffeomorphism on $D^{m}$ or from $M_{2}-\left(M_{2}-D^{m}\right)$ onto $M_{1}-\left(M_{1}-D^{m}\right)$, where for two manifolds $X_{1}$ and $X_{2}, X_{1} \cong X_{2}$ means that $X_{1}$ and $X_{2}$ are diffeomorphic.

$$
\begin{aligned}
& \left(M_{1}-\operatorname{Int} V_{1}\right) \bigcup_{\Phi}\left(M_{2}-\operatorname{Int} V_{2}\right) \\
\cong & \left(M_{1}-\operatorname{Int} V_{1}\right) \bigcup_{\Phi}\left(\left(D^{m}-\operatorname{Int} V_{2}\right) \bigcup\left(M_{2}-\operatorname{Int} D^{m}\right)\right) \\
\cong & \left(M_{1}-\operatorname{Int} V_{1}\right) \bigcup_{\Phi}\left(\left(S^{m}-\left(\operatorname{Int} V_{2} \sqcup \operatorname{Int} D^{m}\right)\right) \bigcup_{\Psi}\left(M_{2}-\operatorname{Int} D^{m}\right)\right) \\
\cong & \left(M_{1}-\operatorname{Int} D^{m}\right) \bigcup_{\Psi}\left(M_{2}-\operatorname{Int} D^{m}\right)
\end{aligned}
$$

This means that the resulting manifold, which is the connected sum of $M_{1}$ and $M_{2}$, admits a round fold map into $\mathbf{R}^{n}$. More precisely, the resulting map is obtained by gluing two maps $\left.f_{1}\right|_{M_{1}-\operatorname{Int} V_{1}}$ and $\left.f_{2}\right|_{M_{2}-\operatorname{Int} V_{2}}$ and satisfies all the conditions mentioned in Proposition 3.

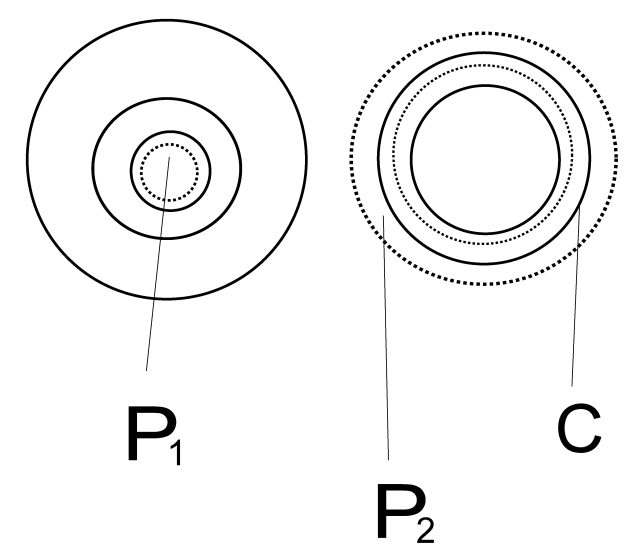

FIGURE 2. $P_{1}, P_{2}, C \subset \mathbf{R}^{n}$ ( $P_{1}$ is the bounded region bounded by the dotted line in the left figure and $P_{2}$ is the region bounded by the disjoint union of the two dotted lines in the right figure.) 
An oriented manifold in a class of $\Theta_{(m, n)}$ admits a round fold map whose singular set is connected into $\mathbf{R}^{n}$, which satisfies all the conditions mentioned in Proposition 3 and a manifold having the structure of an $S^{m-n}$-bundle over $S^{n}$ admits a round fold map into $\mathbf{R}^{n}$ satisfying all the conditions mentioned in Proposition 3 such that the singular set consists of two connected components as mentioned in Remark 1.

By the induction, we obtain a round fold map satisfying all the conditions in Proposition 3 on any manifold $M$ represented as a connected sum of a finite number of $m$-dimensional closed manifolds having the structures of $S^{m-n}$-bundles over $S^{n}$ and an oriented manifold in a class of $\Theta_{(m, n)}$.

We easily have the following proposition.

PROposition 4. Let $M$ be a closed manifold of dimension $m$ and let $f: M \rightarrow \mathbf{R}^{n}$ be a locally $C^{\infty}$ trivial round fold map. Let $F \neq \varnothing$ be a closed manifold and $M^{\prime}$ be a closed manifold having the structure of an $F$-bundle such that for any connected component $C$ of $f(S(f))$ and a small closed tubular neighborhood $N(C)$ of $C$ as in Definition 5, the restriction to $f^{-1}(N(C))$ is a trivial bundle. Then on $M^{\prime}$, there exists a locally $C^{\infty}$ trivial round fold map $f^{\prime}: M^{\prime} \rightarrow \mathbf{R}^{n}$.

Proof. We may assume that $f: M \rightarrow \mathbf{R}^{n}$ is a normal form. Let $S(f)$ consist of $l$ connected components. Set $P_{0}:=D^{n}{ }_{\frac{1}{2}}$ and $P_{k}:=D_{k+\frac{1}{2}}^{n}-\operatorname{Int} D_{k-\frac{1}{2}}^{n}$ for an integer $1 \leq k \leq l$. Then, $f^{-1}\left(P_{k}\right)$ has the structures of trivial bundles over $\partial D_{k-\frac{1}{2}}^{n}$ and $\partial D_{k+\frac{1}{2}}^{n}$ with fibers diffeomorphic to a compact manifold, which we denote by $E_{k}$, such that $\left.f\right|_{f^{-1}\left(\partial D^{n}{ }_{k-\frac{1}{2}}\right)}$ and $\left.f\right|_{f^{-1}\left(\partial D^{n}{ }_{k+\frac{1}{2}}\right)}$ give the structures of subbundles (we denote fibers of two subbundles by $E_{k}{ }^{1} \subset E_{k}$ and $E_{k}{ }^{2} \subset E_{k}$, respectively) for $1 \leq k \leq l$. For any integer $1 \leq k \leq l$ and a diffeomorphism $\phi_{k}$ from $f^{-1}\left(\partial D_{k-\frac{1}{2}}^{n}\right) \subset f^{-1}\left(P_{k}\right)$ onto $f^{-1}\left(\partial D_{k-\frac{1}{2}}^{n}\right) \subset f^{-1}\left(P_{k-1}\right)$ regarded as a bundle isomorphism between the two trivial bundles over standard spheres inducing the identification between the base spaces, $M$ is regarded as $\left(\cdots\left(\left(f^{-1}\left(D^{n} \frac{1}{2}\right)\right) \bigcup_{\phi_{1}} f^{-1}\left(P_{1}\right)\right) \cdots\right) \bigcup_{\phi_{l}} f^{-1}\left(P_{l}\right)$ and for a diffeomorphism $\Phi_{k}$ which is regarded as a bundle isomorphism between the two trivial $F$-bundles inducing the identification $\phi_{k}$ of the base spaces, $M^{\prime}$, which has the structure of an $F$-bundle over $M$, is regarded as $\left(\cdots\left(\left(f^{-1}\left(D^{n}{ }_{\frac{1}{2}}\right) \times F\right) \bigcup_{\Phi_{1}}\left(f^{-1}\left(P_{1}\right) \times F\right)\right) \cdots\right) \bigcup_{\Phi_{l}}\left(f^{-1}\left(P_{l}\right) \times F\right)$. We construct a map on $f^{-1}\left(P_{k}\right) \times F$. This manifold has the structures of trivial bundles over $\partial D_{k-\frac{1}{2}}^{n}$ and $\partial D_{k+\frac{1}{2}}^{n}$ with fibers diffeomorphic to $E_{k} \times F$. On $E_{k} \times F$ there exists a Morse function $\tilde{f}_{k}$ such that the followings hold.

1. $\tilde{f}_{k}\left(E_{k} \times F\right) \subset\left[k-\frac{1}{2}, k+\frac{1}{2}\right]$ and $\tilde{f}_{k}\left(\operatorname{Int}\left(E_{k} \times F\right)\right) \subset\left(k-\frac{1}{2}, k+\frac{1}{2}\right)$ hold.

2. $\tilde{f}_{k}\left(E_{k}{ }^{1} \times F\right)=\left\{k-\frac{1}{2}\right\}$ holds if $E_{k}{ }^{1} \times F$ is non-empty.

3. $\tilde{f}_{k}\left(E_{k}^{2} \times F\right)=\left\{k+\frac{1}{2}\right\}$ holds if $E_{k}^{2} \times F$ is non-empty. 
4. Singular points of $\tilde{f}_{k}$ are in the interior of $E_{k} \times F$ and at two distinct singular points, the values are always distinct.

We obtain a map $\operatorname{id}_{S^{n-1}} \times \tilde{f}_{k}: S^{n-1} \times E_{k} \times F \rightarrow S^{n-1} \times\left[k-\frac{1}{2}, k+\frac{1}{2}\right]$. We can identify $S^{n-1} \times\left[k-\frac{1}{2}, k+\frac{1}{2}\right]$ with $P_{k}=D_{k+\frac{1}{2}}^{n}-\operatorname{Int} D_{k-\frac{1}{2}}^{n}$ by identifying $(p, t) \in S^{n-1} \times\left[k-\frac{1}{2}, k+\frac{1}{2}\right]$ with $t p \in P_{k}$, where we regard $S^{n-1}$ as the unit sphere of dimension $n-1$. By gluing the map $\left.f\right|_{f^{-1}\left(D^{n} \frac{1}{2}\right)} \times \operatorname{id}_{F}$ and the family $\left\{\operatorname{id}_{S^{n-1}} \times \tilde{f}_{k}\right\}$ together by using the family $\left\{\Phi_{k}\right\}$ and the family of identifications in the target manifold $\mathbf{R}^{n}$, we obtain a new round fold map $f^{\prime}: M^{\prime} \rightarrow \mathbf{R}^{n}$.

In the proof, from $f: M \rightarrow \mathbf{R}^{n}$, we obtain $f^{\prime}: M^{\prime} \rightarrow \mathbf{R}^{n}$. We call the operation of constructing $f^{\prime}$ from $f$ a $P$-operation by $F$ on $f$.

As a special case, we obtain the following theorem immediately.

THEOREM 2. In the situation of Proposition 4 , let $F \neq \emptyset$ be a closed manifold and let $p \in M$. Let $M^{\prime}$ be a closed manifold having the structure of an $F$-bundle whose restriction to $M-\{p\}$ is trivial. Then, by a P-operation by $F$, we can construct a round fold map $f^{\prime}: M^{\prime} \rightarrow \mathbf{R}^{n}$.

REMARK 2. In the situation of Theorem 2, for any point $p^{\prime} \in M$, the restriction of an $F$-bundle over $M$ to $M-\left\{p^{\prime}\right\}$ is trivial.

EXAMPLE 1. 1. If a smooth sphere $M$ of dimension $m$ admits a locally $C^{\infty}$ trivial round fold map $f: M \rightarrow \mathbf{R}^{n}(m \geq n \geq 2)$, then for any closed manifold $F$ and any closed manifold $M^{\prime}$ having the structure of an $F$-bundle over $M$, we obtain a round fold map $f^{\prime}: M^{\prime} \rightarrow \mathbf{R}^{n}$ by a P-operation by $F$ on $f$.

For example, every almost-sphere of dimension $m \geq 2$ admits a locally $C^{\infty}$ trivial round fold map into $\mathbf{R}^{2}$ whose singular set is connected.

Some $m$-dimensional smooth spheres admit the structures of $S^{m-n}$-bundles over $S^{n}(m>n \geq 2)$. For example, $S^{3}$ has the structure of an $S^{1}$-bundle over $S^{2}$ and the standard 7-dimensional sphere $S^{7}$ and some 7-dimensional smooth spheres that are not diffeomorphic to $S^{7}$ have the structures of $S^{3}$-bundles over $S^{4}$ (see also [4] and [14]). On such $m$-dimensional spheres, we can construct a round fold map into $\mathbf{R}^{n}$ as mentioned in Remark 1 .

We may apply Theorem 2 to the round fold maps.

2. A connected manifold $X$ of dimension $k \geq 0$ is said to be almost-parallelizable if the restriction of the tangent bundle $T X$ over $X$ to $X-\{p\}(p \in X)$ is trivial. Smooth spheres are of course almost-parallelizable.

The bundle $T X$ over $X$ is a linear bundle whose structure group is $O(k)$. We can define a unit tangent bundle as a natural associated linear bundle whose fiber is $\partial D^{k}=S^{k-1} \subset D^{k}$. 
Let $M$ be a closed, connected and almost-parallelizable manifold of dimension $m$, let $M$ admit a locally $C^{\infty}$ trivial round fold map $f: M \rightarrow \mathbf{R}^{n}(m \geq n \geq 2)$. Let $M^{\prime}$ be a unit tangent bundle of $M$. On $M^{\prime}$, we can construct a round fold map into $\mathbf{R}^{n}$ by a P-operation by $S^{m-1}$ on $f$.

For example, closed manifolds represented as connected sums of products of two spheres are always almost-parallelizable. Proposition 3 states that every closed manifold of dimension $m$ represented as a connected sum of finite copies of $S^{n} \times S^{m-n}$ with $n \geq 2$ and $m \geq 2 n$ assumed admits a locally $C^{\infty}$ trivial round fold map into $\mathbf{R}^{n}$ such that regular fibers are disjoint unions of finite copies of $S^{m-n}$ and that the number of connected components of the singular set and the number of connected components of the fiber of a point in a proper core agree. We may apply Theorem 2 to the round fold map.

4.3. Round fold maps on manifolds having the structures of $S^{1}$-bundles. In this subsection, we denote the $k$-th (co)homology group of a topological space $X$ whose coefficient ring is $R$ by $H_{l}(X ; R)\left(\operatorname{resp} . H^{l}(X ; R)\right)$.

We recall fundamental terms and known facts on $S^{1}$-bundles. It is well-known that $S^{1}$ bundles are regarded as linear bundles whose structure groups are $O(2)$. An $S^{1}$-bundle is said to be orientable if the structure group is regarded as $S O(2)$. Since $S^{1}$-bundles are linear bundles, so we can consider their Stiefel-Whitney classes, which are cohomology classes of the base spaces, where the coefficient rings are $\mathbf{Z} / 2 \mathbf{Z}$, including their 1st Stiefel-Whitney classes, which are 1st cohomology classes of the base spaces. If an $S^{1}$-bundle is orientable, then we can orient the bundle and consider its Euler class, which is a 2nd cohomology class of the base space, where the coefficient ring is $\mathbf{Z}$. We introduce known facts on classifications of linear $S^{1}$-bundles without proofs.

Proposition 5. Let $X$ be a topological space.

1. The 1 st Stiefel-Whitney class $\alpha \in H^{1}(X ; \mathbf{Z} / 2 \mathbf{Z})$ of a linear $S^{1}$-bundle over $X$ vanishes if and only if the bundle is orientable.

2. For any $\alpha \in H^{2}(X ; \mathbf{Z})$, there exists a linear oriented $S^{1}$-bundle over $X$ whose Euler class is $\alpha$.

3. Two linear oriented $S^{1}$-bundles over $X$ are isomorphic if the Euler classes are same.

For general theory of linear (vector) bundles and their characteristic classes including StiefelWhitney classes and Euler classes, see also [17] for example.

We have the following.

THEOREM 3. Let $M$ be a closed manifold of dimension $m$, let $f: M \rightarrow \mathbf{R}^{n}$ be a locally $C^{\infty}$ trivial round fold map and let $m \geq n \geq 3$. Suppose either of the followings.

1. $n \geq 4$ holds.

2. $n=3$ holds, $f(M)$ is diffeomorphic to $D^{3}$ and $M$ is connected. 
Assume that for any connected component $C$ of $f(S(f))$, a normal fiber $F_{C}$ of $C$ corresponding to a trivial bundle over $C$ as in Definition 5 satisfies $H^{2}\left(F_{C} ; \mathbf{Z}\right) \cong\{0\}$. Then, for any closed manifold $M^{\prime}$ having the structure of an orientable $S^{1}$-bundle over $M$, by a P-operation by $S^{1}$ on $f$, we can obtain a round fold map $f^{\prime}: M^{\prime} \rightarrow \mathbf{R}^{n}$.

Furthermore, for any connected component $C$ of $f(S(f))$, let the fiber $F_{C}$ above satisfy $H^{1}\left(F_{C} ; \mathbf{Z} / 2 \mathbf{Z}\right) \cong\{0\}$. Then, for any closed manifold $M^{\prime}$ having the structure of an $S^{1}-$ bundle over $M$, by a P-operation by $S^{1}$ on $f$, we can obtain a locally $C^{\infty}$ trivial round fold map $f^{\prime}: M^{\prime} \rightarrow \mathbf{R}^{n}$.

PROOF. First we prove statements in the case where $n \geq 4$ holds.

For any connected component $C$ of $f(S(f))$, there exist a small closed tubular neighborhood $N(C)$ and a trivial bundle $f^{-1}(N(C))$ over $C$ as in Definition 5 and we may regard that $H^{2}\left(f^{-1}(N(C)) ; \mathbf{Z}\right) \cong H^{2}\left(C \times F_{C} ; \mathbf{Z}\right) \cong H^{2}\left(F_{C} ; \mathbf{Z}\right) \cong\{0\}$ holds since by the assumption, for a normal fiber $F_{C}$ corresponding to the bundle as in Definition $5, H^{2}\left(F_{C} ; \mathbf{Z}\right) \cong\{0\}$ is assumed. If we restrict any orientable $S^{1}$-bundle over $M$ to $f^{-1}(N(C))$, then the bundle obtained by the restriction is a trivial bundle since the Euler class vanishes if the obtained bundle is oriented. We can do a P-operation by $S^{1}$ on $f$.

Assume that $H^{1}\left(F_{C} ; \mathbf{Z} / 2 \mathbf{Z}\right) \cong\{0\}$ holds. $H^{1}\left(f^{-1}(N(C)) ; \mathbf{Z} / 2 \mathbf{Z}\right) \cong H^{1}(C \times$ $\left.F_{C} ; \mathbf{Z} / 2 \mathbf{Z}\right) \cong\{0\}$ holds since $H^{1}\left(F_{C} ; \mathbf{Z} / 2 \mathbf{Z}\right) \cong\{0\}$ holds. If we restrict any $S^{1}$-bundle over $M$ to $f^{-1}(N(C))$, then the bundle obtained by the restriction is orientable since the 1 st Stiefel-Whitney class vanishes. As a result, the obtained bundle is a trivial bundle by the previous discussion on the Euler class. We can do a P-operation similarly.

Thus, this completes the proof of the statements in the case where $n \geq 4$ holds. We prove statements in the latter case.

For any connected component $C$ of $f(S(f))$, there exist a small closed tubular neighborhood $N(C)$ and a trivial bundle $f^{-1}(N(C))$ over $C$ as in Definition 5 and $H^{2}\left(f^{-1}(N(C)) ; \mathbf{Z}\right) \cong H^{2}\left(C \times F_{C} ; \mathbf{Z}\right) \cong H^{0}\left(F_{C} ; \mathbf{Z}\right) \otimes H^{2}(C ; \mathbf{Z}) \cong H^{0}\left(F_{C} ; \mathbf{Z}\right) \otimes \mathbf{Z}$ holds since $H^{2}\left(F_{C} ; \mathbf{Z}\right) \cong\{0\}$ is assumed.

For any connected component $C$ of $f(S(f))$ and a connected component $E_{C}{ }^{\lambda}$ of $f^{-1}(N(C))$, if the restriction of an orientable $S^{1}$-bundle over $M$ to a connected component of $f^{-1}(\partial N(C)) \bigcap E_{C}{ }^{\lambda}$ is orientable and the Euler class of the obtained (oriented) bundle vanishes, then the restriction of the bundle over $M$ to $E_{C}{ }^{\lambda}$ is orientable and the Euler class of the obtained (oriented) bundle vanishes since $H^{2}\left(F_{C} ; \mathbf{Z}\right) \cong\{0\}$ is assumed. The restriction of the bundle over $M$ to $f^{-1}(\partial N(C)) \bigcap E_{C}{ }^{\lambda}$ is also orientable and the Euler class of the (oriented) bundle obtained by this restriction vanishes by the same reason.

Let $F$ be the fiber of a point in a proper core $P$ of $f$. Note that for a proper core $P$ of $f$, which is diffeomorphic to $D^{3}, f^{-1}(P) \neq \emptyset$ has the structure of a trivial $F$-bundle over $P$. $f^{-1}(\partial P)$ is regarded as the restriction of the bundle $f^{-1}(P)$ to the boundary $\partial P$ of a proper core $P$ and for the connected component $C_{0}$ of $f(S(f))$ in the center and the given small closed tubular neighborhood $N\left(C_{0}\right)$, it is regarded as a subbundle of the trivial bundle 
$f^{-1}\left(N\left(C_{0}\right)\right)$ over $C_{0}$. The fiber of the bundle $f^{-1}\left(N\left(C_{0}\right)\right)$ is diffeomorphic to $F_{C_{0}}$ satisfying $H^{2}\left(F_{C_{0}} ; \mathbf{Z}\right) \cong\{0\}$. Hence, the Euler class of the restriction of the bundle over $M$ to any connected component of $f^{-1}(\partial P)$ vanishes if the obtained bundle is oriented.

Since $M$ and the Reeb space $W_{f}$ of the map $f: M \rightarrow \mathbf{R}^{n}$ are connected, by these discussions and the induction, it follows that for any connected component $C$ of $f(S(f))$, the Euler class of the restriction of an oriented $S^{1}$-bundle over $M$ to $f^{-1}(N(C))$ vanishes. We can do a P-operation by $S^{1}$ on $f$.

Assume that $H^{1}\left(F_{C} ; \mathbf{Z} / 2 \mathbf{Z}\right) \cong\{0\}$ also holds. We note that $H^{1}\left(f^{-1}(N(C)) ; \mathbf{Z} / 2 \mathbf{Z}\right) \cong$ $\{0\}$ holds since $H^{1}\left(F_{C} ; \mathbf{Z} / 2 \mathbf{Z}\right) \cong\{0\}$ is assumed. By the argument similar to that of the proof of the case where $n \geq 4$ holds, if we restrict any $S^{1}$-bundle over $M$ to $f^{-1}(N(C))$, then the bundle obtained by the restriction is orientable and as a result it is a trivial $S^{1}$-bundle.

Thus this completes the proof of the theorem.

We have the following theorem in the case where the target manifold is $\mathbf{R}^{2}$.

THEOREM 4. Let $M$ be a closed manifold of dimension $m \geq 2$ and $f: M \rightarrow \mathbf{R}^{2}$ be a locally $C^{\infty}$ trivial round fold map.

1. For any connected component $C$ of $f(S(f))$, we denote a small closed tubular neighborhood of $C$ as in Definition 5 by $N(C)$ and we denote a normal fiber of $C$ corresponding to the trivial bundle $f^{-1}(N(C))$ over $C$ as in Definition 5 by $F_{C}$. Let $F_{C}$ satisfy $H^{1}\left(F_{C} ; \mathbf{Z}\right) \cong H^{1}\left(F_{C} ; \mathbf{Z} / 2 \mathbf{Z}\right) \cong H^{2}\left(F_{C} ; \mathbf{Z}\right) \cong\{0\}$. Then by a P-operation by $S^{1}$ on $f$, for any closed manifold $M^{\prime}$ having the structure of an $S^{1}$-bundle over $M$ such that for any connected component $C$ of $f(S(f))$, the restriction to $f^{-1}(\partial N(C))$ is an orientable bundle, we can obtain a round fold map $f^{\prime}: M^{\prime} \rightarrow \mathbf{R}^{2}$.

2. Furthermore, if $f(M)$ is diffeomorphic to $D^{2}$ and $M$ is connected, then by a $P$ operation by $S^{1}$ on $f$, for any closed manifold $M^{\prime}$ having the structure of an $S^{1}$ bundle over $M$, we can obtain a round fold map $f^{\prime}: M^{\prime} \rightarrow \mathbf{R}^{2}$.

ProOF. We prove the first part. For any connected component $C$ of $f(S(f))$, $H^{2}\left(f^{-1}(N(C)) ; \mathbf{Z}\right) \cong H^{2}\left(C \times F_{C} ; \mathbf{Z}\right) \cong\{0\}$ holds since $H^{1}\left(F_{C} ; \mathbf{Z}\right) \cong H^{2}\left(F_{C} ; \mathbf{Z}\right) \cong$ $H^{2}(C ; \mathbf{Z}) \cong\{0\}$ holds. If we restrict any $S^{1}$-bundle over $M$ to $f^{-1}(N(C))$ such that the restriction to $f^{-1}(\partial N(C))$ is an orientable $S^{1}$-bundle, then the bundle obtained by the restriction is orientable since $H^{1}\left(f^{-1}(N(C)) ; \mathbf{Z} / 2 \mathbf{Z}\right) \cong H^{1}\left(C \times F_{C} ; \mathbf{Z} / 2 \mathbf{Z}\right) \cong H^{1}(C ; \mathbf{Z} / 2 \mathbf{Z}) \cong \mathbf{Z} / 2 \mathbf{Z}$ holds and the 1 st Stiefel-Whitney class of the obtained bundle vanishes and as a result, it is a trivial bundle since $H^{2}\left(f^{-1}(N(C)) ; \mathbf{Z}\right) \cong H^{2}\left(C \times F_{C} ; \mathbf{Z}\right) \cong\{0\}$ holds. By a P-operation by $S^{1}$ on $f$, a round fold map $f^{\prime}: M^{\prime} \rightarrow \mathbf{R}^{2}$ is obtained.

This completes the proof of the first part. Now we prove the second part.

For any connected component $C$ of $f(S(f))$ and any connected component $E_{C}{ }^{\lambda}$ of $f^{-1}(N(C))$, if the restriction of the given $S^{1}$-bundle $M^{\prime}$ over $M$ to a connected component of $\partial E_{C}{ }^{\lambda}$ is orientable and the Euler class of the obtained (oriented) bundle vanishes, then 
the restriction of the bundle over $M$ to $E_{C}{ }^{\lambda}$ is orientable and the Euler class of the obtained (oriented) bundle vanishes by the discussions on 1st Stiefel-Whitney classes and Euler classes above. The restriction of the bundle over $M$ to $f^{-1}(\partial N(C)) \bigcap E_{C}{ }^{\lambda} \neq \emptyset$ is also orientable and the Euler class of the bundle obtained by this restriction vanishes by the same reason.

Let $F$ be the fiber of a point in a proper core $P$ of $f$. Note that for a proper core $P$ of $f, f^{-1}(P) \neq \emptyset$ has the structure of a trivial $F$-bundle over $P . f^{-1}(\partial P)$ is regarded as the restriction of the bundle $f^{-1}(P)$ to $\partial P$ and for the connected component $C_{0}$ of $f(S(f))$ in the center and the given small closed tubular neighborhood $N\left(C_{0}\right)$, it is regarded as a subbundle of the trivial bundle $f^{-1}\left(N\left(C_{0}\right)\right)$. The fiber of the bundle $f^{-1}\left(N\left(C_{0}\right)\right)$ is diffeomorphic to $F_{C_{0}}$ satisfying $H^{1}\left(F_{C_{0}} ; \mathbf{Z}\right) \cong H^{1}\left(F_{C_{0}} ; \mathbf{Z} / 2 \mathbf{Z}\right) \cong H^{2}\left(F_{C_{0}} ; \mathbf{Z}\right) \cong\{0\}$. By the discussions here, the 1 st Stiefel-Whitney class of the restriction of any $S^{1}$-bundle over $M$ to $f^{-1}(\partial P)$ vanishes and the Euler class of this restriction of the bundle, which is defined by orienting the obtained bundle, vanishes.

Since $M$ and the Reeb space $W_{f}$ of the map $f: M \rightarrow \mathbf{R}^{n}$ are connected, by the arguments above and the induction, for any connected component $C$ of $f(S(f))$, the restriction of any $C^{\infty} S^{1}$-bundle over $M$ to $f^{-1}(N(C))$ is orientable and the Euler class of the resulting (oriented) bundle vanishes.

Thus this completes the second part of the proof and this completes the proof.

We consider a round fold map $f: M \rightarrow \mathbf{R}^{n}$ presented in Proposition 3 ( $n \geq 2$ and $m \geq 2 n$ hold). For any connected component $C$ of $f(S(f))$ and a small closed tubular neighborhood $N(C)$ of $C, f^{-1}(N(C))$ has the structure of a trivial bundle over $C$ as in Definition 5 and for a normal fiber $F_{C}$ of $C$ corresponding to the bundle $f^{-1}(N(C))$, $H^{1}\left(F_{C} ; \mathbf{Z}\right) \cong H^{2}\left(F_{C} ; \mathbf{Z}\right) \cong\{0\}$ holds if $m \geq 5$ holds. Thus, $f$ satisfies the assumptions of all the statements of Theorem 3 or all the statements of Theorem 4 in the case where $m \geq 5$ holds.

In this situation, if $n \geq 3$ holds, then $H^{2}(M ; \mathbf{Z}) \cong\{0\}$ holds and $S^{1}$-bundles over $M$ are always trivial. In the following example, we study topological properties of $S^{1}$-bundles over $M$ in the case where $n=2$ holds.

EXAMPLE 2. 1. Let $M$ be a closed manifold of dimension $m \geq 5$ diffeomorphic to a manifold having the structure of an $S^{m-2}$-bundle over $S^{2}$. Any closed manifold $M^{\prime}$ having the structure of an orientable $S^{1}$-bundle over $M$ has the structure of an $S^{m-2}$ bundle over a 3-dimensional manifold having the structure of an $S^{1}$-bundle over $S^{2}$. We study the topological properties of such manifolds and apply P-operations by $S^{1}$ on round fold maps on the manifolds into the plane.

Note that for a generator $v$ of the cohomology group $H^{2}(M ; \mathbf{Z}) \cong \mathbf{Z}$ and for any integer $k$, there exists a closed manifold $M^{\prime}{ }_{k}$ having the structure of an oriented $S^{1}$ bundle over $M$ whose Euler class is $k v \in H^{2}(M ; \mathbf{Z})$. Note also that $H^{m-2}(M ; \mathbf{Z}) \cong$ $\mathbf{Z}$ holds and that $H^{l}(M ; \mathbf{Z}) \cong\{0\}$ holds for $l \neq 0,2, m-2, m$. We can consider the following well-known exact sequence (Gysin sequence); $p_{k}: M^{\prime}{ }_{k} \rightarrow M$ denotes the 
projection of the $S^{1}$-bundle $M^{\prime}{ }_{k}$ over $M$ and $\bigcup k v$ denotes the operation of taking cup products with $k v$ (for such a sequence, see [17] for example).

$$
\begin{aligned}
\cdots \longrightarrow H^{m-2}(M ; \mathbf{Z}) \cong \mathbf{Z} \stackrel{\bigcup k v}{\longrightarrow} H^{m}(M ; \mathbf{Z}) \cong \mathbf{Z} \\
\stackrel{p_{k}{ }^{\prime}}{\longrightarrow} H^{m}\left(M^{\prime}{ }_{k} ; \mathbf{Z}\right) \longrightarrow H^{m-1}(M ; \mathbf{Z}) \cong\{0\}
\end{aligned}
$$

By virtue of Poincare duality theorem, we have $H_{1}\left(M^{\prime}{ }_{k} ; \mathbf{Z}\right) \cong H^{m}\left(M^{\prime}{ }_{k} ; \mathbf{Z}\right)$ and the group $H_{1}\left(M^{\prime}{ }_{k} ; \mathbf{Z}\right)$ is isomorphic to $\mathbf{Z}$ for $k=0$, is isomorphic to $\{0\}$ for $|k|=1$ and is isomorphic to $\mathbf{Z} /|k| \mathbf{Z}$ for $|k| \geq 2$.

We obtain a new round fold map into $\mathbf{R}^{2}$ on $M^{\prime}\left(M^{\prime}{ }_{k}\right)$ by a P-operation by $S^{1}$ on a round fold map $f: M \rightarrow \mathbf{R}^{2}$ presented in Proposition 3 .

2. Let $M$ be a closed manifold of dimension $m \geq 5$ represented as the connected sum of two closed oriented manifolds $M_{1}$ and $M_{2}$ having the structures of $S^{m-2}$-bundles over $S^{2}$.

$M$ is obtained by gluing the two manifolds $M_{1}-\operatorname{Int} D^{m}$ and $M_{2}-\operatorname{Int} D^{m}$ by a diffeomorphism between the boundaries. Let $v_{i} \in H^{2}\left(M_{i}-\operatorname{Int} D^{m} ; \mathbf{Z}\right) \cong \mathbf{Z}$ be a generator of the cohomology group for $i=1$, 2. Clearly $H^{2}(M ; \mathbf{Z}) \cong H^{2}\left(M_{1}-\right.$ $\left.\operatorname{Int} D^{m} ; \mathbf{Z}\right) \oplus H^{2}\left(M_{2}-\operatorname{Int} D^{m} ; \mathbf{Z}\right) \cong \mathbf{Z} \oplus \mathbf{Z}$ holds. Note also that $H^{m-2}(M ; \mathbf{Z}) \cong$ $H^{m-2}\left(M_{1}-\operatorname{Int} D^{m} ; \mathbf{Z}\right) \oplus H^{m-2}\left(M_{2}-\operatorname{Int} D^{m} ; \mathbf{Z}\right) \cong \mathbf{Z} \oplus \mathbf{Z}$ holds and that $H^{l}(M ; \mathbf{Z}) \cong$ $\{0\}$ holds for $l \neq 0,2, m-2, m$.

For any integer $k$, there exists a closed manifold $M^{\prime}{ }_{k, 0}$ having the structure of an oriented $S^{1}$-bundle over $M$ whose Euler class is $\left(k v_{1}, 0\right) \in H^{2}(M ; \mathbf{Z}) \cong H^{2}\left(M_{1}-\right.$ $\left.\operatorname{Int} D^{m} ; \mathbf{Z}\right) \oplus H^{2}\left(M_{2}-\operatorname{Int} D^{m} ; \mathbf{Z}\right) \cong \mathbf{Z} \oplus \mathbf{Z}$. As in the previous discussion, we consider the following sequence; $p_{k, 0}: M^{\prime}{ }_{k} \rightarrow M$ denotes the projection of the $S^{1}$ bundle $M^{\prime}{ }_{k, 0}$ over $M$ and $\left(k v_{1}, 0\right)$ denotes the operation of taking cup products with $\left(k v_{1}, 0\right)$.

$$
\begin{aligned}
& \cdots \longrightarrow H^{m-2}(M ; \mathbf{Z}) \cong \mathbf{Z} \oplus \mathbf{Z} \stackrel{\cup\left(k v_{1}, 0\right)}{\longrightarrow} H^{m}(M ; \mathbf{Z}) \cong \mathbf{Z} \\
& \stackrel{p_{k, 0^{*}}}{\longrightarrow} H^{m}\left(M_{k, 0}^{\prime} ; \mathbf{Z}\right) \longrightarrow H^{m-1}(M ; \mathbf{Z}) \cong\{0\}
\end{aligned}
$$

By virtue of Poincare duality theorem, we have $H_{1}\left(M^{\prime}{ }_{k, 0} ; \mathbf{Z}\right) \cong H^{m}\left(M^{\prime}{ }_{k, 0} ; \mathbf{Z}\right)$ and the group $H_{1}\left(M_{k, 0}^{\prime} ; \mathbf{Z}\right)$ is isomorphic to $\mathbf{Z}$ for $k=0$, is isomorphic to $\{0\}$ for $|k|=1$ and is isomorphic to $\mathbf{Z} /|k| \mathbf{Z}$ for $|k| \geq 2$.

Generally, for any pair of two integers $\left(k_{1}, k_{2}\right)$, there exists a closed manifold $M^{\prime}{ }_{k_{1}, k_{2}}$ having the structure of an oriented $S^{1}$-bundle over $M$ whose Euler class is $\left(k_{1} v_{1}, k_{2} v_{2}\right) \in H^{2}(M ; \mathbf{Z}) \cong H^{2}\left(M_{1}-\operatorname{Int} D^{m} ; \mathbf{Z}\right) \oplus H^{2}\left(M_{2}-\operatorname{Int} D^{m} ; \mathbf{Z}\right) \cong \mathbf{Z} \oplus \mathbf{Z}$.

On the manifolds $M^{\prime}{ }_{k, 0}$ and $M^{\prime}{ }_{k_{1}, k_{2}}$ presented here, we can construct new round fold maps by P-operations by $S^{1}$ on a round fold map $f: M \rightarrow \mathbf{R}^{2}$ presented in Proposition 3. 
We note about the homeomorphism and diffeomorphism types of the resulting source manifolds.

In this situation, let $M_{1}:=S^{2} \times S^{m-2}$ and let $M_{2}$ be a closed manifold having the structure of a linear $S^{m-2}$-bundle over $S^{2}$ which is not trivial. $M_{1}$ is spin and $M_{2}$ is not spin. Thus, $M^{\prime}{ }_{k, 0}$ is not spin and $M_{0,1}^{\prime}$ is spin. In fact, the diffeomorphism types of these manifolds are as the followings.

(a) The manifold $M^{\prime}{ }_{k, 0}$ is given by gluing a manifold diffeomorphic to the product of $S^{m-2}$ and a 3-dimensional manifold having the structure of an oriented $S^{1}$-bundle over $S^{2}$ with the interior of a submanifold diffeomorphic to $S^{1} \times D^{m}$ removed and a manifold diffeomorphic to the product of $M_{2}$ and $S^{1}$ with the interior of a submanifold diffeomorphic to $S^{1} \times D^{m}$ removed together by a diffeomorphism between the boundaries.

(b) The manifold $M^{\prime}{ }_{0,1}$ is given by gluing a manifold diffeomorphic to the product of two manifolds $S^{2} \times S^{m-n}-\operatorname{Int} D^{m}$ and $S^{1}$ and a manifold having the structure of $S^{m-2}$-bundle over $S^{3}$ with the interior of a submanifold diffeomorphic to $S^{1} \times D^{m}$ removed together by a diffeomorphism between the boundaries.

As a result, it follows that $M_{k, 0}^{\prime}$ and $M^{\prime}{ }_{0,1}$ are not homeomorphic. Moreover, we have the following exact sequence as before; we denote the projection of the $S^{1}$ bundle $M^{\prime}{ }_{k_{1}, k_{2}}$ over $M$ by $p_{k_{1}, k_{2}}$ and by $\bigcup\left(k_{1} v_{1}, k_{2} v_{2}\right)$ the operation of taking cup products with $\left(k_{1} v_{1}, k_{2} v_{2}\right)$ as before.

$$
\begin{aligned}
& \cdots \quad H^{l}(M ; \mathbf{Z}) \quad \stackrel{\bigcup\left(k_{1} v_{1}, k_{2} v_{2}\right)}{\longrightarrow} H^{l+2}(M ; \mathbf{Z}) \cong \mathbf{Z} \\
& \stackrel{p_{k_{1}, k_{2}}{ }^{*}}{\longrightarrow} H^{l+2}\left(M^{\prime} k_{1}, k_{2} ; \mathbf{Z}\right) \longrightarrow H^{l+1}(M ; \mathbf{Z}) \stackrel{\bigcup\left(k_{1} v_{1}, k_{2} v_{2}\right)}{\longrightarrow} \ldots
\end{aligned}
$$

From the exact sequence, we have that the (co)homology groups of the two manifolds $M_{1,0}^{\prime}$ and $M_{0,1}^{\prime}$ are isomorphic.

REMARK 3. The 5-dimensional real projective space $\mathbf{R} P^{5}$ admits an orientable $S^{1}$ bundle structure over the 2-dimensional complex projective space $\mathbf{C} P^{2}$. It is known that $\mathbf{C} P^{2}$ does not admit any fold map into $\mathbf{R}^{n}$ for $2 \leq n \leq 4$. It is known that $\mathbf{R} P^{5}$ admits a fold map into $\mathbf{R}^{2}$ and does not admit any fold map into $\mathbf{R}^{3}$ but we do not know if $\mathbf{R} P^{5}$ admits a fold map into $\mathbf{R}^{4}$. For existence problems for fold maps on manifolds, see also [1], [6], [7], [18] and [19] for example.

The facts above mean that we cannot obtain a round fold map from $\mathbf{R} P^{5}$ into $\mathbf{R}^{n}$ by a P-operation by $S^{1}$ on a round fold map from $\mathbf{C} P^{2}(2 \leq n \leq 4)$.

\section{References}

[1] Y. ANDo, Folding maps and the surgery theory on manifolds, J. Math. Soc. Japan Volume 53, Number 2 (2001), 357-382. 
[ 2 ] D. BArden, Simply Connected Five-Manifolds, Ann. of Math. (3) 82 (1965), 365-385.

[ 3 ] O. Burlet, G. DE Rham, Sur certaines applications generiques d'une variete close a 3 dimensions dans le plan, Enseign. Math. 20 (1974), 275-292.

[ 4 ] J. J. Eells and N. H. KuIPER, An invariant for certain smooth manifolds, Ann. Mat. Pura Appl. 60 (1962), 93-110.

[ 5 ] C. Ehresmann, Les connexions infinitesimales dans un espace fibre differentiable, Colloque de Topologie, Bruxelles (1950), 29-55.

[ 6 ] Y. Eliashberg, On singularities of folding type, Math. USSR Izv. 4 (1970), 1119-1134.

[ 7 ] Y. Eliashberg, Surgery of singularities of smooth mappings, Math. USSR Izv. 6 (1972), 1302-1326.

[ 8 ] Y. K. S. FURUYA and P. PorTo, On special generic maps from a closed manifold into the plane, Topology Appl. 35 (1990), 41-52.

[9] M. Golubitsky and V. Guillemin, Stable Mappings and Their Singularities, Graduate Texts in Mathematics (14), Springer-Verlag (1974).

[10] N. KitaZaWA, On round fold maps (in Japanese), RIMS Kokyuroku Bessatsu B38 (2013), 45-59.

[11] N. KitAZAWA, Fold maps with singular value sets of concentric spheres, to appear in Hokkaido Mathematical Journal.

[12] N. KiTAZAWA, Differential topology of manifolds admitting round fold maps, arXiv:1304.0618 (2013).

[13] M. Kobayashi and O. SAeKi, Simplifying stable mappings into the plane from a global viewpoint, Trans. Amer. Math. Soc. 348 (1996), 2607-2636.

[14] J. W. MiLnor, On manifolds homeomorphic to the 7-sphere, Ann. of Math. (2) 64 (1956), 399-405.

[15] J. Milnor, Morse theory, Ann. of Math. AM-51, Princeton University Press; 1st Edition (1963).

[16] J. Milnor, Lectures on the h-cobordism theorem, Math. Notes, Princeton Univ. Press, Princeton, N. J. (1965).

[17] J. Milnor and J. StashefF, Characteristic classes, Annals of Mathematics Studies, No. 76. Princeton, N. J; Princeton University Press (1974).

[18] R. SAdy Kov, O. SAEKI and K. SAKuma, Obstructions to the existence of fold maps, J. London Math. Soc. (2) 81 (2010), 338-354.

[19] O. SAEKI, Notes on the topology of folds, J. Math. Soc. Japan Volume 44, Number 3 (1992), 551-566.

[20] O. SAEKI, Topology of special generic maps of manifolds into Euclidean spaces, Topology Appl. 49 (1993), 265-293.

[21] O. Saeki and K. Sakuma, On special generic maps into $\mathbf{R}^{3}$, Pacific J. Math. 184 (1998), 175-193.

[22] K. SAKUMA, On special generic maps of simply connected $2 n$-manifolds into $\mathbf{R}^{3}$, Topology Appl. $\mathbf{5 0}$ (1993), 249-261.

[23] N. Steenrod, The topology of fibre bundles, Princeton University Press (1951).

[24] R. Тном, Les singularites des applications differentiables, Ann. Inst. Fourier (Grenoble) 6 (1955-56), $43-87$.

[25] H. Whitney, On singularities of mappings of Euclidean spaces: I, mappings of the plane into the plane, Ann. of Math. 62 (1955), 374-410.

Present Address:

DEPARTMENT OF MATHEMATICS,

TOKYO INSTITUTE OF TECHNOLOGY,

2-12-1 Ookayama, Meguro-KU, TOKyo 152-8551, JAPAN.

e-mail: kitazawa.n.aa@m.titech.ac.jp 